CASE REPORT

\author{
P.T.L. Chiam \\ R.M. Samuelson \\ J. Mocco \\ R.A. Hanel \\ A.H. Siddiqui \\ L.N. Hopkins \\ E.I. Levy
}

\title{
Navigability Trumps All: Stenting of Acute Middle Cerebral Artery Occlusions with a New Self- Expandable Stent
}

\begin{abstract}
SUMmARY: Acute stroke intervention is rapidly evolving. New technologies are improving device deliverability and rates of recanalization. We describe 2 cases of acute middle cerebral artery occlusions wherein Wingspan stents could not be delivered to the occlusive site because of excessive vascular tortuosity. Merci thrombectomy was also unsuccessful. Revascularization was only achieved with deployment of the highly navigable Enterprise stent, resulting in thrombolysis in myocardial infarction 2/3 flow. Thus, all devices should be considered in the armamentarium of stroke therapy.
\end{abstract}

ntra-arterial therapies for acute stroke have evolved rapidly in recent years. ${ }^{1,2}$ Although the newer-generation Merci mechanical clot retriever (Concentric Medical, Mountain View, Calif) achieved successful recanalization in $70 \%$ of patients, ${ }^{2}$ this was only marginally superior to the $66 \%$ recanalization rate in the Prolyse in Acute Cerebral Thromboembolism II trial. ${ }^{1}$ Although encouraging, substantial room for improvement exists.

Coronary (balloon-expandable) stents have been used successfully in acute stroke intervention with published recanalization rates of $79 \% .{ }^{3}$ Use of a balloon- or self-expandable stent has been found to be an independent predictor of successful recanalization (OR, 4.8). ${ }^{4}$ Modern balloon-expandable stents have become increasingly trackable and flexible; however, compared with self-expandable stents specifically designed for the intracranial circulation, balloon-expandable stents are larger and stiffer. ${ }^{5}$

On the basis of early, encouraging results of acute intracranial revascularization with stents and knowledge that self-expandable stents are more trackable and have lower profiles, a 2-center registry, Stent Assisted Recanalization in Ischemic Stroke Phase I Study to Evaluate the Safety of Wingspan Stenting (SARIS), is currently enrolling patients by using the Wingspan stent (Boston Scientific, Natick,

\section{Received April 17, 2008; accepted after revision May 20}

From the Department of Neurosurgery and Toshiba Stroke Research Center (P.T.L.C., R.M.S., J.M., A.H.S., L.N.H., E.I.L.), Department of Radiology (A.H.S., L.N.H., E.I.L.), School of Medicine and Biomedical Sciences, University at Buffalo, State University of New York, Buffalo, NY; Department of Neurosurgery (P.T.L.C., R.M.S., J.M., A.H.S., L.N.H., E.I.L.), Millard Fillmore Gates Hospital, Kaleida Health, Buffalo, NY; and Department of Neurosurgery (R.A.H.), Mayo Clinic, Jacksonville, Fla.

Dr. Hanel serves as a consultant to NeuroVasx and on the speaker's bureau for Cordis. Dr. Hopkins receives research support from Boston Scientific, Cordis, and Micrus; and honoraria from Bard, Boston Scientific, and Cordis; has an ownership interest in AccessClosure, Boston Scientific, Micrus, and Square One; and serves as a consultant to/member of the advisory board for Abbott, Bard, Boston Scientific, Cordis, and Micrus. Dr. Levy receives research support, other research support (devices), and honoraria from Boston Scientific; receives research support from ev3; serves as a consultant to Cordis Neurovascular and Micrus Endovascular; and receives patent royalties from Zimmer Spine and fees for carotid stent training from Abbott Vascular and ev3. Dr. Mocco has received a research grant from the Brain Aneurysm Foundation. Dr. Siddiqui has received a research grant from the University at Buffalo and honoraria from Genentech.

Please address correspondence to Elad I. Levy, MD, Department of Neurosurgery and Toshiba Stroke Research Center, University at Buffalo, State University of New York, 3 Gates Circle, Buffalo, NY 14209; e-mail: elevy@buffns.com

DOI 10.3174/ajnr.A1221
Mass) for acute stroke intervention. We report 2 cases wherein the highly navigable Wingspan could not be delivered to occlusive sites because of excessive vascular tortuosity, and, after unsuccessful Merci thrombectomy, Enterprise self-expandable stents (Cordis, Miami Lakes, Fla) were delivered successfully to acutely occluded cerebral vessels with immediate restoration of flow.

\section{Case Reports}

\section{Case 1 Description}

A 67-year-old woman with diabetes mellitus, hypertension, and dyslipidemia was admitted with sudden onset of aphasia and right-sided weakness of 5 hours' duration. (Fig 1) The National Institutes of Health Stroke Scale (NIHSS) score was 25. Head CT demonstrated no hemorrhage; CT perfusion was consistent with significant left middle cerebral artery territory ischemia without significant infarcted tissue. $\mathrm{CT}$ angiography revealed distal left $\mathrm{M} 1$ occlusion and severe looping of the left cervical internal carotid artery (ICA).

Informed consent was obtained for participation in SARIS. The patient was intubated and brought to the angiography suite.

Via right femoral artery access, a 5F Vitek catheter (Cook, Bloomington, Ind) was advanced to the left common carotid artery (LCCA). A stiff exchange-length 0.035-inch Glidewire (Terumo, Tokyo, Japan) was advanced to the distal LCCA. The Vitek was replaced with a 6F Neuron guide catheter (Penumbra, Alameda, Calif), which was placed in the cervical segment after moderate manipulation because of severe LICA tortuosity. A Gold Tip 0.016-inch wire (Terumo) and a Nautica microcatheter (ev3, Irvine, Calif) were advanced across the occlusion. The Gold Tip was exchanged for a Transend-300 wire (Boston Scientific), and the Nautica was removed.

A $3.0-\mathrm{mm} \times 15-\mathrm{mm}$ Wingspan was then advanced over the Transend but could not be maneuvered beyond the proximal left M1 despite multiple attempts, owing to cervical ICA and intracranial circulation tortuosity, with "backing out" of the Neuron. Repeat attempts after positioning the Neuron in the distal cervical ICA also proved futile. Recognizing that more guide support was required, we removed all equipment. An $8 \mathrm{~F}$ Simmons-2 guide catheter (Cordis, Warren, NJ) was placed into the LCCA. For additional support and to avoid catheter prolapse, a V18 wire (Boston Scientific) was placed through the guide catheter into the left external carotid artery. A Synchro ${ }^{2}$ Standard wire (Boston Scientific) and the Nautica were then advanced across the occlusion. The microcatheter was removed and attempts at advancing the Wingspan to the occlusion were again un- 

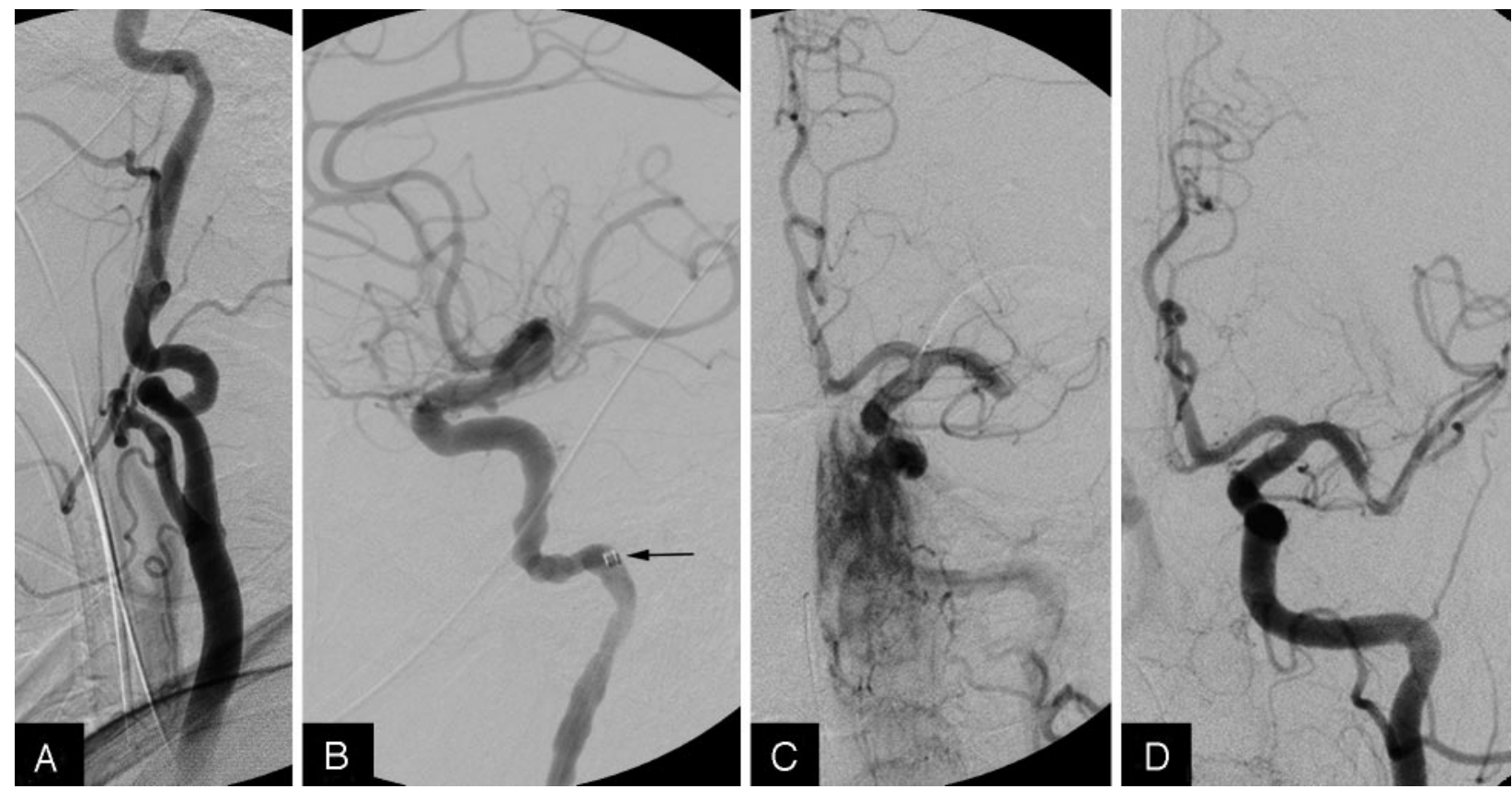

Fig 1. A, Angiogram showing severe tortuosity of the left ICA. $B$, Neuron guide catheter (arrow) positioned deep in the ICA. $C$, Intracranial angiogram showing distal M1 occlusion. $D$, Recanalization of the M1 immediately after stent placement.
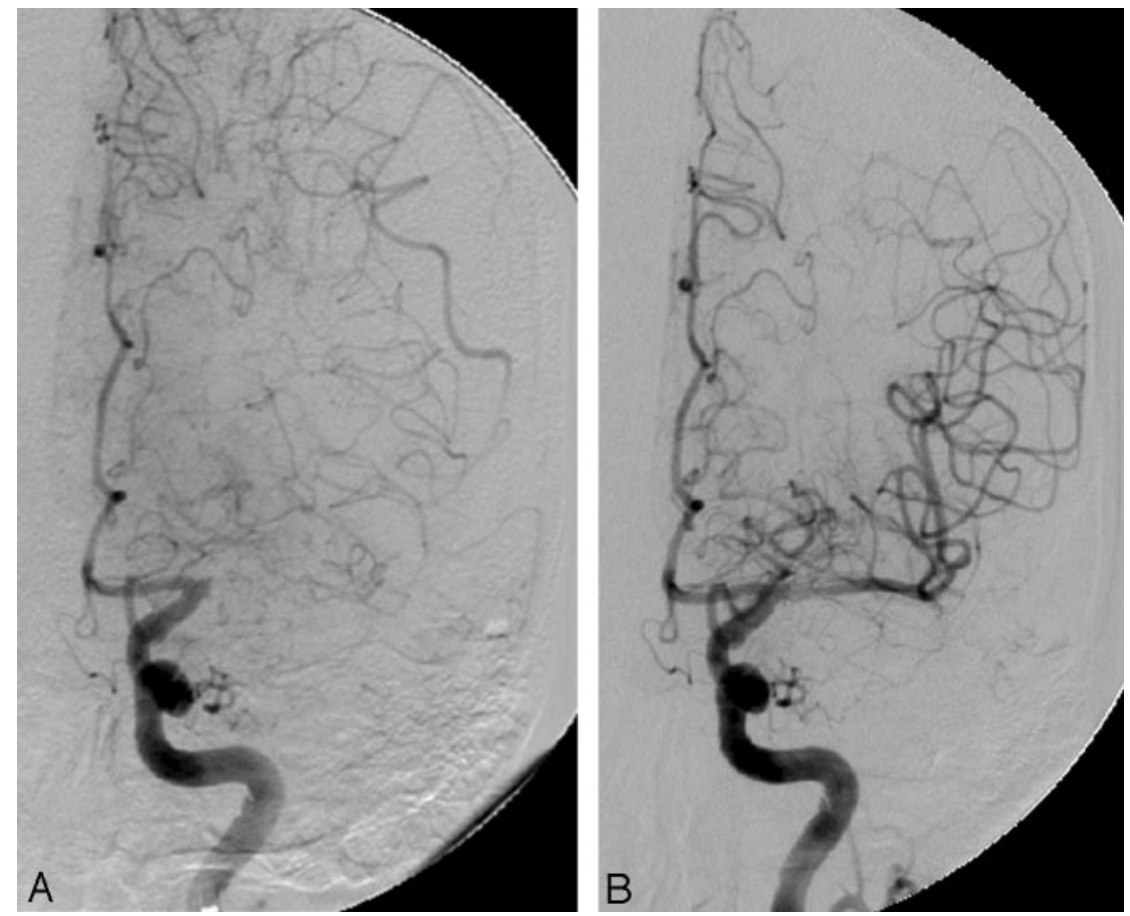

Fig 2. A, Angiogram showing tortuosity of the cavernous ICA and occlusion of the proximal M1. B, Recanalization of the M1 immediately after stent placement.

successful. The Nautica was then replaced with a Merci $18 \mathrm{~L}$ microcatheter, which was placed distal to the occlusion. A Merci L5 clot retriever was deployed, but clot retrieval was unsuccessful.

As a "last resort," an Enterprise was used in an off-label fashion to attempt recanalization. The Merci $18 \mathrm{~L}$ microcatheter was exchanged for a Prowler Select Plus microcatheter (Cordis). A $4.5-\mathrm{mm} \times 22-\mathrm{mm}$ Enterprise was then advanced through the microcatheter to the occlusion. This stent crossed the occlusion without significant difficulty. After optimal positioning, the stent was fully deployed. Partial deployment followed by stent recovery was considered; however, a partially occlusive thrombus remained near the proximal stent edge. Angiograms showed immediate restoration of Thrombolysis in Myocardial Infarction (TIMI) 2 flow with recanalization of the inferior M2. Because it was already 7 hours from symptom onset, the procedure was terminated because of decreasing benefit-risk ratio.

No hemorrhage was seen on postprocedure CT. The patient was extubated on day 3 with an NIHSS of 15 at 2 weeks. 


\section{Case 2 Summary}

An 85-year-old woman with a history of atrial fibrillation was admitted with sudden onset of right-sided weakness and dysphasia of 2 hours' duration (Fig 2). NIHSS score was 16; head CT showed no hemorrhage. Intravenous tissue plasminogen activator (tPA) was commenced (Interventional Management of Stroke protocol). Repeat angiogram 1 hour later showed persistent proximal left M1 occlusion. Intra-arterial tPA and Merci thrombectomy failed to recanalize the vessel. A 4.0-mm $\times 20$-mm Wingspan could not be delivered to the occlusion; finally, recanalization was successful with use of $4.5-\mathrm{mm} \times 22-\mathrm{mm}$ Enterprise. NIHSS score was 1 at 48 hours postprocedure.

\section{Discussion}

Use of balloon-expandable or self-expandable stents in acute stroke is feasible, with a successful recanalization rate (TIMI $2 / 3$ ) of $79 \% .^{3,6}$ Stent placement in this setting may minimize the need for additional thrombolytic therapy with its attendant risks. In a previous animal model study, both the Liberte (Boston Scientific) balloon-expandable and Wingspan selfexpandable stents were equally effective in reestablishing flow through acutely occluded vessels. ${ }^{7}$ Self-expandable stents were superior with respect to navigability and incurred lower rates of vasospasm and side-branch occlusion. An additional potential benefit is that they do not require balloon inflation in vessels whose true diameter and degree of atherosclerosis are unclear.

Because of this superiority, the Wingspan is currently being investigated in a 2-center registry approved by the US Food and Drug Administration (FDA) to determine its effectiveness and safety in acutely occluded intracranial arteries. In both cases reported here, the Wingspan could not be maneuvered to target locations because of tortuous and unfavorable anatomy. After failure of Merci retrieval, an Enterprise was used as a "last resort" measure.

The Enterprise is a closed-cell, self-expandable stent currently approved by the FDA for use in stent-assisted coiling of intracranial aneurysms. Although self-expandable like the Wingspan, it has a different delivery system design, allowing for superior navigability. This difference proved to be the critical technical factor because Enterprise stents were delivered to occlusion sites without significant problems. After significant procedural times in attempting to maneuver Wingspan stents to the target sites and unsuccessful Merci thrombectomies, Enterprise stents were delivered and deployed rapidly, with achievement of TIMI 2/3 flow. A recent report described the use of the Enterprise deployed temporarily and recaptured in an acute stroke, without the concerns of permanent stent implantation. ${ }^{8}$ We considered partial deployment followed by stent recovery; however, the decision to fully deploy the stent was made because near-occlusive thrombus remained after partial deployment.

These reports strongly emphasize consideration of all available devices in the armamentarium for acute stroke therapy. Additional studies are required to assess efficacy and safety of this stent in acutely occluded intracranial vessels so that patients can benefit from this technology.

\section{Conclusion}

Acute stroke intervention is rapidly evolving, with new technologies improving deliverability of devices and, thus, recanalization rates. We describe 2 cases wherein the Wingspan could not be delivered to the vessel occlusion site and successful recanalization was only achieved with the highly navigable Enterprise.

\section{Acknowledgments}

We thank Paul H. Dressel for preparation of the illustrations.

\section{References}

1. Furlan A, Higashida R, Wechsler L, et al. Intra-arterial prourokinase for acute ischemic stroke. The PROACT II study: a randomized controlled trial. Prolyse in Acute Cerebral Thromboembolism. JAMA 1999;282:2003-11

2. Smith WS, Sung G, Saver J, et al. Mechanical thrombectomy for acute ischemic stroke: final results of the Multi MERCI trial. Stroke 2008;39:1205-12

3. Levy EI, Ecker RD, Horowitz MB, et al. Stent-assisted intracranial recanalization for acute stroke: early results. Neurosurgery 2006;58:458-63; discussion $58-63$

4. Gupta R, Vora NA, Horowitz MB, et al. Multimodal reperfusion therapy for acute ischemic stroke: factors predicting vessel recanalization. Stroke 2006;37:986-90

5. Migliavacca F, Petrini L, Massarotti P, et al. Stainless and shape memory alloy coronary stents: a computational study on the interaction with the vascular wall. Biomech Model Mechanobiol 2004;2:205-17

6. Levy EI, Mehta R, Gupta R, et al. Self-expanding stents for recanalization of acute cerebrovascular occlusions. AJNR Am J Neuroradiol 2007;28:816-22

7. Levy EI, Sauvageau E, Hanel RA, et al. Self-expanding versus balloon-mounted stents for vessel recanalization following embolic occlusion in the canine model: technical feasibility study. AJNR Am J Neuroradiol 2006;27:2069-72

8. Kelly ME, Furlan AJ, Fiorella D. Recanalization of an acute middle cerebra artery occlusion using a self-expanding, reconstrainable, intracranial microstent as a temporary endovascular bypass. Stroke 2008;39:1770-73 